\title{
The Neurologist in the Emergency Room: Prospective Study of the Neurological Emergency Group in Lombardia
}

MariaLuisa Delodovici ${ }^{1}$, Marco Mauri ${ }^{1}$, Megi Meneri ${ }^{1}$, Marco Gallazzi ${ }^{1}$, Elena Ballabio ${ }^{2}$, Giuseppina Borutti ${ }^{3}$, Maria Vittoria Calloni ${ }^{4}$, Elisa Candeloro ${ }^{5}$, Bruno Censori ${ }^{6}$, Claudio De Piazza ${ }^{7}$, Laura Fusi ${ }^{8}$, Franca Mazzucchelli ${ }^{9}$, Ignazio Santilli ${ }^{10}$, Elisabetta Forapani ${ }^{10}$, Chiara Scaccabarozzi ${ }^{11}$, Maria Sessa ${ }^{12}$, Lucia Tancredi ${ }^{13}$ and Massimiliano Braga ${ }^{14}$

1. Dept. of Neurology and Stroke Unit, Ospedale di Circolo e Fondazione Macchi, Università dell'Insubria, ASST-Settelaghi, le Luigi Borri 5721100 Varese, Italy

2. Dept. of Neurology, Ospedale S. Carlo Borromeo, A. Mosca 1220152 Milano, Italy

3. Dept. of Neurology, Director Ospedale Civile di Voghera, Volturno 14 Voghera 27058 (PV), Italy

4. Dept. of Neurology, Ospedale di Legnano ASST Ovest Milanese, Papa Giovanni Paolo II, 20025 Legnano (MI), Italy

5. Dept of Neurology Fondazione IRCCS C. Mondino, Mondino 2, Pavia 27100, Italy

6. Dept of Neurology ASST Papa Giovanni XXIII, Piazza OMS 124127 Bergamo, Italy

7. Dept. of Neurology Ospedale Valduce, Como, Dante Alighieri 1122100 Como, Italy

8. Dept. of Neurology Ospedale di Saronno, ASST Valle olona, Piazzale Borella 1 Saronno 21047 (VA), Italy

9. Dept of Neurology Ospedale S. Antonio Abate Gallarate. Pastori 421013 Gallarate, Varese, Italy

10. Dept. of Neurology Ospedale di Desio (MB) Director, G. Mazzini 120832 Desio (MB), Italy

11. Dept. of Neurology ASST, Lecco-Ospedale A. Manzoni, dell'Eremo 9/11 23900 Lecco, Italy

12. Dept. of Neurology Ospedale di Cremona, ASST Cremona Director, le Concordia 126100 Cremona, Italy

13. Dept. of Neurology Ospedale Sant'Anna, Como, Ravona 2022042 S, Fermo della Battaglia, Como, Italy

14. Dept. of Neurology Ospedale di Vimercate (MB), Santi Cosma e Damiano 1020871 Vimercate (MB), Italy

\begin{abstract}
Purpose of the work was to quantify the workloads and the neurologist's commitment in ER, to verify the management of urgencies and appropriateness of neurological counseling requests. Neurologic emergencies are frequent in elderly patients and are associated with high morbidity and mortality. Due to the aging population, neurologic pathologies in emergency room (ER) are widely expected to increase. The role of the neurologist in this context is controversial. Methods: in 14 operative units of neurology a prospective collection of neurological evaluations in ER was carried out using boards of detection of the most frequent neurological clinical pictures seen in the course of a week. Data were collected from 738 cases. Attention was focused on cerebrovascular diseases (CVD), impairment of consciousness, epilepsy, headache, dizziness, head trauma and disorders of the peripheral nervous system (PNS). Achievements: neurological consultations were on average $5 \%$ of total accesses to ER. The most common pathology seen was ischemic stroke. After counseling most patients were discharged home. The CVD required the greatest commitment to the specialist. The usefulness of the specialized neurological "filter" has been confirmed in a non-simple operative context, aimed at optimizing the appropriateness of the hospitalization and the economy of the diagnostic path.
\end{abstract}

Key words: Emergency room, neurological diseases, neurology, workload. 


\section{Introduction}

Given an aging population, the care of the elderly patient in the emergency room (ER) is becoming increasingly common [1]. Neurologic emergencies are frequent in elderly patients and are associated with high morbidity and mortality. In this context distinguished symptoms related to internal medical conditions from those of neurological diseases are often really challenging. A recent published report showed that the presence of a neurologist in ER reduced the length of stay, brain imaging number and hospital admission rate for neurologic patients [2]; however other older reports showed contradictory results concerning hospitalization rate for patients receiving specialist consultation, the use of advanced imaging and length of stay [3]. Commonly neurological emergency situations are reported mainly in the context of CVD, head trauma and seizures, conditions that may present high mortality and costs arising from acute management and long-term care. In many of these conditions is crucial for the good patient outcome that therapy is initiated rapidly [4, 5]. As underlined by current guidelines the presence of neurologists in the ER is of highest importance [6, 7]. A recent paper has underlined that the ER is a high-risk site for diagnostic errors and sought to estimate ER diagnostic accuracy for identifying acute cerebrovascular events [8]. A nationwide survey performed in Italy [9] in 2003 provided useful information concerning the burden of neurological acute diseases and the role of neurologists in ER, obtaining data similar to those from other European countries $[6,7,9]$ and suggesting the careful attention of healthcare administrators; in this survey CVD was the main cause of neurological consultations (27\%), followed by headache (22\%), dizziness (13\%), head trauma (13\%), impairment of consciousness (12\%) and epilepsy (9\%). In order to assess the current role of

Corresponding author: MariaLuisa Delodovici, M.D., head of Stroke Unit, research fields: cerebrovascular disease and neurophysiology. the neurologist in ER we undertook a prospective multicenter study involving territorial hospitals of Lombardia. The aim of this study was to evaluate the profile of patients referred to ER, to quantify workloads and commitment of the neurologist in this context, to document the usefulness of the specialized "filter" for the percentage and appropriateness of the hospitalization, to compare the initial diagnosis of the requesting physician with the diagnosis at discharge and to verify the contents of the demand for neurological performance in order to identify and propose indicators of appropriateness and quality.

\section{Materials and Methods}

The study was conducted in 14 hospitals located in Lombardia, a region in north-west of Italy during last months of 2017 and first months of 2018. These hospitals are representative of the whole territory being some located in large urban centers and others in rural and mountain areas. All the hospitals had a neurological department with on-duty and on-call neurologists and hospital settings showed the presence of intensive care, emergency medicine wards or departments and Stroke Unit. All about 4 centers had a neurosurgical department. In every hospital a referent neurologist was identified.

All consecutive patients who presented in a week in the ER of these hospitals suspected of suffering neurological diseases were evaluated. We considered as activity indicators the number of patients admitted to ER, the neurological ER consultations and the investigations performed in ER. Information concerning modalities of presentation to ER, the type of event, the presence of various risk factors, the diagnostic workup and the treatments was anonymously collected according to low current privacy. The number of admissions, the confirmation of the diagnosis at admission, and the discrepancies between diagnosis at acceptance and at discharge after neurological consultation were considered.

ER discharge diagnoses were categorized into 8 
subgroups: CVD, impairment of consciousness, epilepsy, headache, dizziness, head trauma, peripheral nervous disease (PND) and others. This classification was made according to previous reports that attributed to these diseases the greatest number of accesses in ER [7]. Given the absolute prevalence of cerebrovascular pathology compared to other conditions a pathology-focused analysis was subsequently conducted in the same hospitals among consultations called from the ED for CVD during thirty consecutive days. Twelve hospitals participated in this survey. We additionally reviewed the total number of patients referred to all ER during the study period and the total number of patients seen during one year in each ER.

Continuous variables were treated with descriptive statistics, using mean and standard deviations. Comparative analysis was performed using ANOVA and chi-square test, and $p$-values $<0.05$ were considered to be statistically significant.

\section{Results}

In the hospitals involved in this survey the number of patients referred to ER was between 60,000 and 67,000 cases/year with a mean number of neurological consultation of 3,250/year. From these data it appears that neurological consultation is requested in about 5\% of patients referred to ER.

The weekly sampling carried out by the 14 centers allowed evaluating 738 incident cases, 352 female and 386 males, mean age 75.2 yrs (52\% male).

A mean of 53 neurologic consultations/week were obtained for each center (8 consultations/day).

CVD was confirmed to be the most frequently encountered condition among neurological emergencies, with a frequency of $27 \%$ (Fig. 1), the same reported at the time from the first national epidemiological survey of the project NEU [9].

Unlike cerebrovascular pathology, headache, dizziness and head trauma were represented to a lesser extent than in the previous survey mentioned above, respectively $11 \%$ and $8 \%$. Over $60 \%$ of cases were reported to the ER through the Territorial System of Emergency Urgency (AREU). Subsequently to the Triage and after evaluation of the emergentist in ER incoming patients suspected of suffering neurological diseases were immediately seen by a neurologist, and emergency therapy was initiated with minimum time delay when needed, expecially in case of CVD. The examinations required in ER were: a number of 472 brain CT scan (64\% of cases), a number 73 electroencephalogram

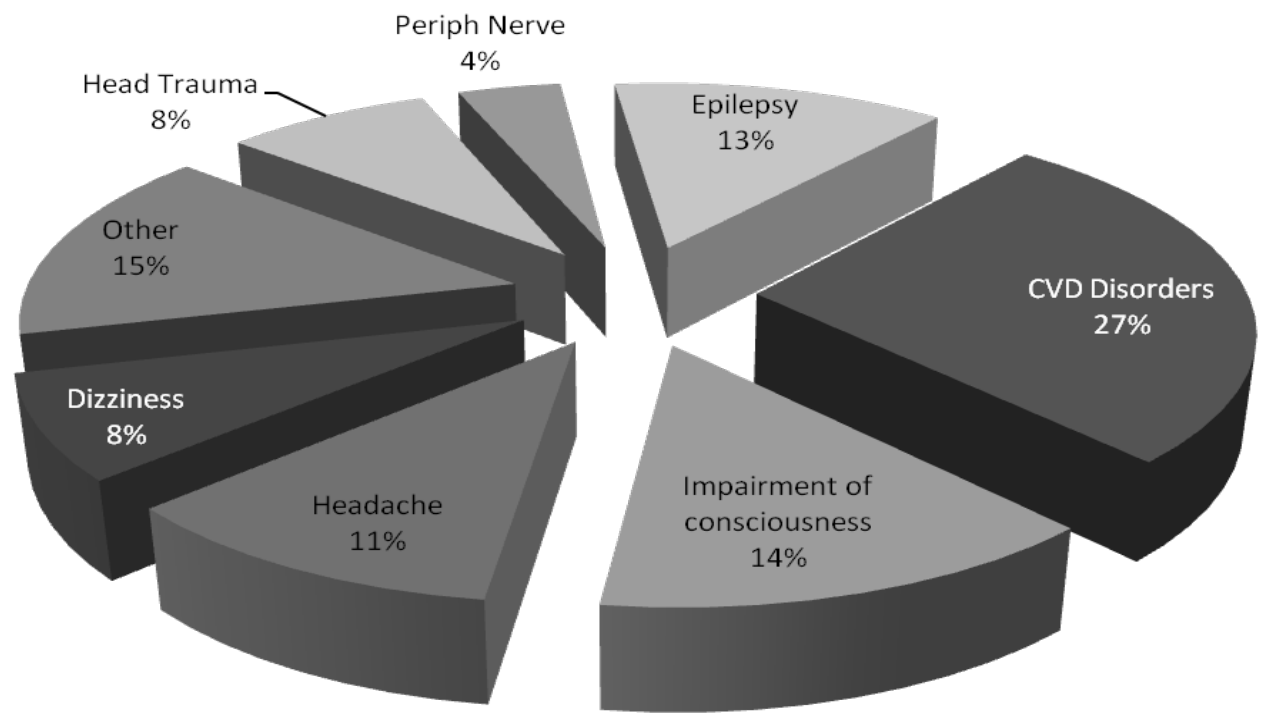

Fig. 1 Commonest clincal conditions requiring neurological consultation in ER during one week. 


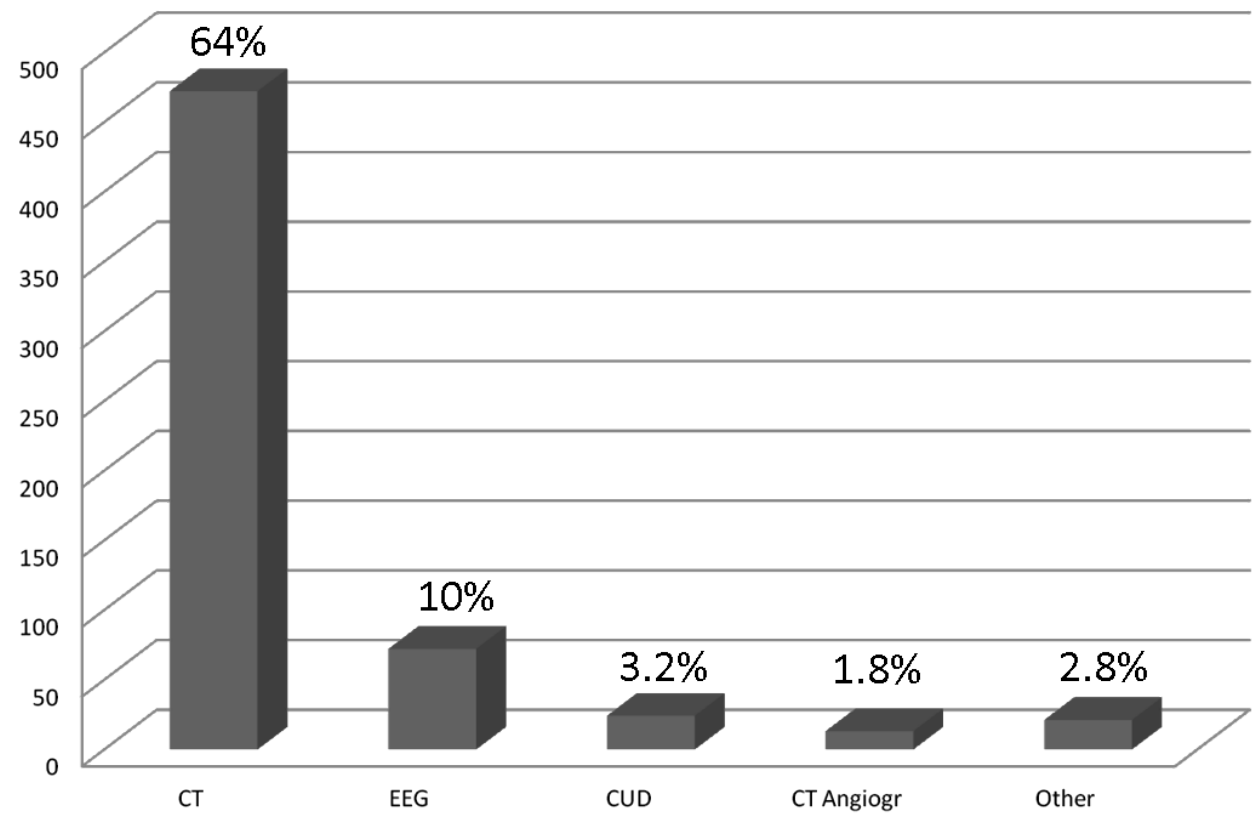

Fig. 2 Diagnostic test performed in ER.

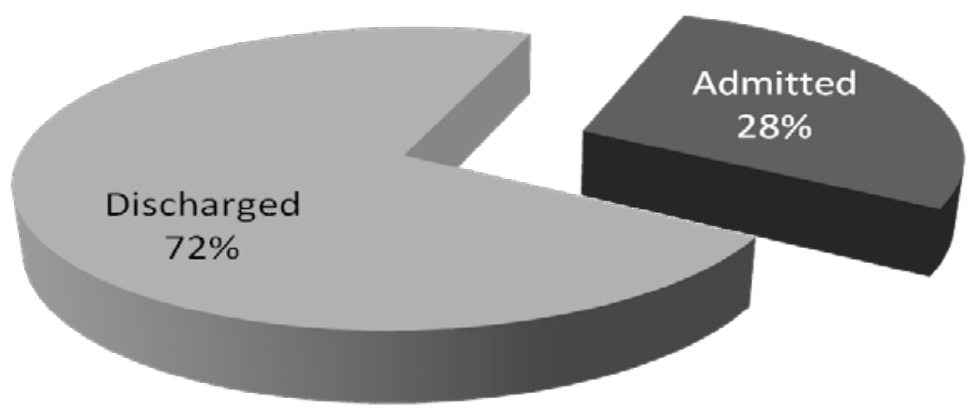

Fig. 3 Patients admitted and discharged after neurological consultation.

(10\%), 24 Carotid Ultrasound Duplex (CUD) (3.2\%), a number of $13 \mathrm{CT}$ angiography (1.8\%), a number of 21 others (2.8\%) (Fig. 2).

After the evaluation of the neurologist $28 \%$ of patients were hospitalized and $72 \%$ discharged home (Fig. 3).

The total number of admission was of 207 patients. The percentage of home discharges was highest in patients with peripheral nerve palsies (97\%), migraine and other headaches (96\%), and trauma (93\%). A different diagnostic approach between emergentist and neurologist was seen in the evaluation of patients with headache; the emergentist performed a CT brain scan in $99 \%$ of the cases; after neurological consultation a CT angiogram or a brain MRI was further performed in order to exclude a symptomatic headache.

Stroke was the most common source of emergency admission to the Neurological Unit (NU), 89\% of cases, followed by $14 \%$ of patients with impairment of consciousness and $8 \%$ of cases with head trauma (Fig. 4).

On this line and on the basis of what is already reported in the literature, given the absolute prevalence of cerebrovascular pathology compared to other conditions, a pathology-focused analysis about patients referred to ER with CVD during thirty days was conducted.

A number of 686 cases with suspected CVD were collected in this 30-day analysis; among this group 576 patients (84\%) were hospitalized and 110 (16\%) discharged from ER after neurological evaluation. 


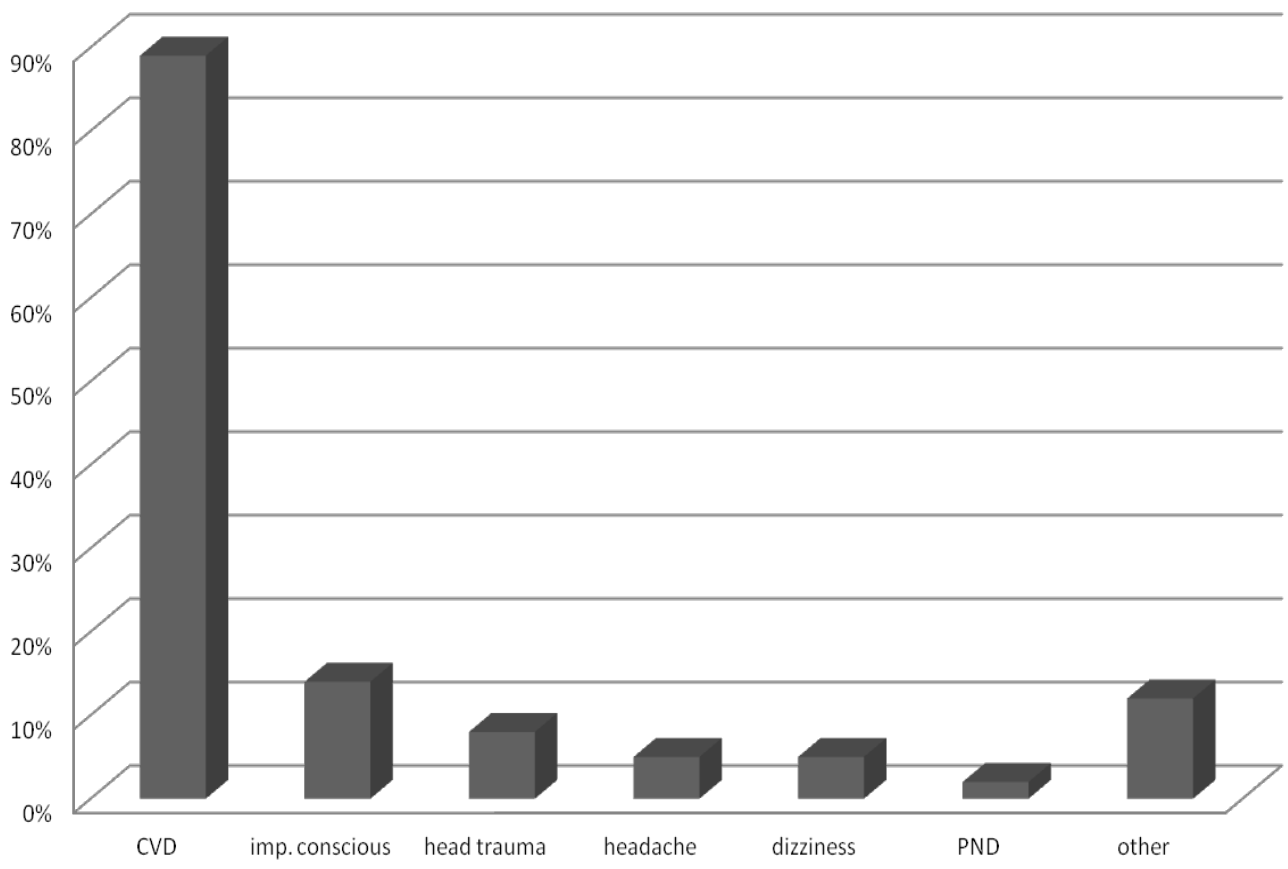

Fig. 4 Percentage of patients admitted to Neurological Unit for each pathology.

Moreover 102 subjects (15\%) discharged from the ER had different diagnosis from the initial one of suspected CVD.

Then considering that in the group of patients dismissed after hospitalization 31 patients (4.5\%) were discharged with a diagnosis other than that of CVD, the number of patients with stroke mimics increased to a total of 133 cases (19.5\%).

The confirmation of a cerebrovascular event revealed ischemic stroke in 343 cases (63\%), Transient Ischemic Attack (TIA) in 125 (23\%) and cerebral hemorrhage in 77 cases (14\%).

Intravenous thrombolysis was performed in $24 \%$ of cases, on average in the most severe cases: in 40 patients (49\%) with NIHSS between 8 and 14, in 13 cases (16\%) with NIHSS $<7$ and in 29 patients with NIHSS $>15$ (35\%) at the ER admission. Mechanical thrombectomy was performed in 27 patients.

All patients encoded as ischemic stroke performed a basal brain CT and 65\% of patients undergoing thrombolytic therapy underwent a CT angiography or a MR angiography.

A number of 50 patients diagnosed with TIA were started in ordinary hospitalization for further investigation. Comparing the diagnostic accuracy of emergentist and neurologist in patients with CVD results in that the role of the neurologist improves the diagnostic definitions of these cases, mainly for patients with TIA $(p<0.05)$. Fig. 5 shows the diagnostic confirmation of cerebrovascular pathology after the neurological evaluation.

The average time devoted by the neurologist to consultations of each patient varied from 20 minutes to 180 minutes (predominantly for patients with CVD). Considering a mean of 8 consultations/day of whom a third dedicated to patients with CVD the average length of neurology consultations in the ED resulted in about 6 hours/day.

Patients with Ischemic Stroke compared to the other acute vascular events required an evaluation/observation time about twice (1.12 h vs. 0.65 h, $p<0.001$ Anova).

In addition, patients undergoing thrombolysis treatments/thrombectomy required an even longer observation/evaluation time compared to patients not undergoing any procedure (2.34 h vs. $0.81 \mathrm{~h}, p<0.001$ Anova). 


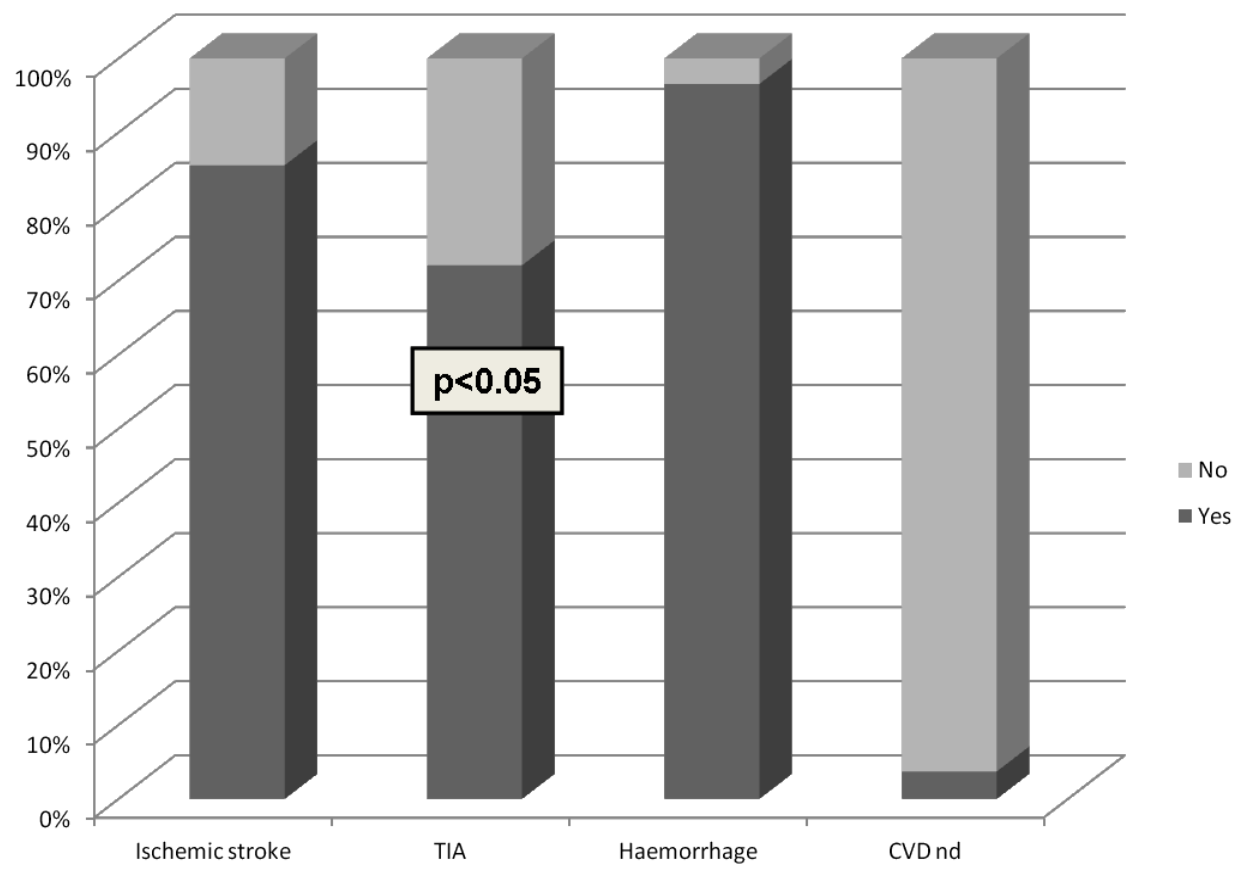

Fig. 5 Confirmation or not of CVD after neurological evaluation.

\section{Discussion}

Neurological urgencies represent a grouping of conditions and diseases, many of which are burdened by high mortality and outcomes often disabling, as in the case of stroke, and others characterized by evolution and prognosis related to the timeliness of diagnosis and treatment, as in the case of seizures, secondary headaches and other acute phenomena [6]. Furthermore elderly patients often have more pathologies and the neurologist has a preminent role in guiding the diagnosis in complicated patients. Several studies document the inappropriateness of many consultations provided in urgency for patients with diseases of the nervous system [10, 11], highlighting the need for a more rational organization and greater appropriateness in diagnostic assessments. Compared to previous work, our report has documented a reduction in neurological counseling for vertigo, head trauma and headaches, and this is probably due to the adoption of management protocols in the ER or to a better outpatient organization for these diseases. The high number of patients arrived with the AREU system documents that it has been effective to discourage the self-presentation of patients in the ER, even if these results can be further improved. The development of medical imaging, together with new possibilities for therapeutic intervention has in recent years changed the approach to Neurologic emergencies, mainly for time-dependent pathology, conferring to the neurologist a prominent role in the diagnosis and treatment activity within ER [12-14]. This manuscript emphasizes the dual role of the neurologist in ER, first of all in the management of urgency, being mainly involved in cerebrovascular pathology. In this case the specialist is to be considered-in particular for CVD, but also for headaches and epilepsy-the Case-manager responsible for the entire diagnostic and therapeutic path during the stay in ER. His role also in differential diagnosis (19\% stroke mimics) must be considered. The time needed to manage the cerebrovascular patient in urgency is often particularly high, so the number of consultations (8/day) does not adequately represent the time spent in ER. In addition, the neurologist plays an important role in dismissing patients (72\% of patients were discharged home) and is useful to define a 
diagnostic workup that will be subsequently continued on an outpatient basis. In the absence of the neurologist, the percentage of wrong diagnoses for cases with pathology of specialist expertise is high, with diagnosis "false positive" in 37.3\% and "false negative" in 36.6\%, respectively [10, 15]. Most recent publications have shown how the management process of care by the neurologist allows the reduction of the costs of benefits, as well as increased appropriateness and efficiency of the process of care even in the medium and long-term evaluation of clinical indicators [16]. Over the past 10 years in particular, the absorption of resources by the area of emergency has grown exponentially, for the increasing development of the techniques of neurodiagnostic, for the progress of therapeutic interventions and for a better organization of care for the critically ill patient [17]. The competence of the neurologist has come to integrate into welfare action with neurovascular intervention on the one hand and with the other neurointensive activity, creating the new profile to a specialist with expertise and cooperations in all fields, defined by the term neurohospitalist, an operator with proven experience in managing not only cerebrovascular diseases but also all the neurological disorders that occur at ER, including cases requiring intensive care $[18,19]$. With this in mind, the American Academy of Neurology (AAN) has organized courses and found resources to organize training courses for the issuance of a specific certification in "critical care and emergency neurology” [20, 21]. When evaluating neurological patients in ER it should be considered an expert triage system to evaluate patients in self-presentation; the operating context is often difficult due to various different clinical emergency situations; clinical evaluation may be difficult in non-syndromic pictures, often with isolated symptoms and associated to high costs for test often normal or negative. Various contributions allowed proposing and validating organizational models of emergency neurology, also proposing the possible indicators of quality and appropriateness [21, 22].

\section{Conclusion}

The most relevant information of our analysis confirms the substantial accuracy of diagnosis of CVD by the neurologist and the correctly identifying of pathology, reducing misdiagnosis or diagnostic uncertainty. Benign neurological conditions such as migraine, syncope and peripheral vertigo are frequently mislabeled as seizure or stroke. Educational strategies for these common conditions in ER could improve diagnostic accuracy and may result in better patient care. As urgent neurological diseases seen in ER are often potentially serious, the role of the neurologist is important for its optimum management. The proportion of patients undergoing thrombolysis procedures, accounting for $24 \%$ of cases of cerebral ischemia confirmed by investigation in ER, underlines the importance of neurologists in management of this condition. The main advantage of this work is the prospective nature, that minimizes recruiting bias, and the representative sample of hospitals in Lombardia and these data can provide holds for interventional public health projects. Nevertheless this work has some limitations: firstly, no direct comparison was made with hospitals without the neurologist, to assess whether the absence of the aforementioned specialist significantly impacts the patient's management. On this aspect, it should be noted that, almost in Lombardia, medium and large hospitals all have a unit of neurology; in the smaller hospitals, the AREU sends fewer patients with serious neurological diseases, so it is difficult to perform a comparison due to the presence of numerous confounding factors. Moreover, the hospitals were not analyzed separately with the neurologist on call and those with the neurologist on duty, and no cost-effectiveness analysis was performed for the role of the neurologist.

\section{Declaration of Conflicting Interests}

There are no conflicts of interest for any of the authors of this paper. 


\section{References}

[1] Sang, B. L., Jae, H. O., Jeong, H. P., Seung, P. C., and Jung, H. W. 2018. "Differences in Youngest-Old, Middle-Old, and Oldest-Old Patients Who Visit the Emergency Department.” Clin Exp Emerg Med. 5 (4): 249-55.

[2] Van der Linden, M. C., van den Brand, C. L., van den Wijngaard, I. R., de Beaufort, R. A. Y., van der Linden, N., and Jellema, K. 2018. "A Dedicated Neurologist at the Emergency Department during Out-of-Office Hours Decreases Patients' Length of Stay and Admission Percentages.” Journal of Neurology 265: 535-41.

[3] Pitts, S. R., Morgan, S. R., Schrager, J. D., and Berger, T. J. 2014. "Emergency Department Resource Use by Supervised Residents vs. Attending Physicians Alone.” JAMA 312: 2394-400.

[4] Rizos, T., Jüttler, E., Sykora, M., Poli, S., and Ringleb, P. A. 2011. "Common Disorders in the Neurological Emergency Room-Experience at a Tertiary Care Hospital.” Eur J Neurol. 18 (3): 430-5.

[5] Hacke, W., Kaste, M., Bluhmki, E., Brozman, M., Dávalos, A., Toni, D., et al. 2008. "Thrombolysis with Alteplase 3 to 4.5 Hours after Acute Ischemic Stroke.” N Engl J Med. 359 (13): 1317-29.

[6] Stone, K. 2009. "When Seconds Count: Tackling Neurological Emergencies.” Lancet Neurol. 8 (8): 702-3.

[7] Micieli, G., De Falco, F. A., Consoli, D., Inzitari, D., Sterzi, R., Toni, D., et al. 2012. "The Role of Emergency Neurology in Italy: Outcome of a Consensus Meeting for a Intersociety Position.” Neurol Sci. 33 (2): 297-304.

[8] Tarnutzer, A. A., Lee, S. H., Robinson, K. A., Wang, Z., Edlow, J. A., and Newman-Toker, D. E. 2017. "ED Misdiagnosis of Cerebrovascular Events in the Era of Modern Neuroimaging: A Meta-Analysis.” Neurology 88 (15): 1468-77.

[9] De Falco, F. A., Sterzi, R., Toso, V., Provinciali, L., Leone, M. A., Beghi, E., et al. 2008. "The Neurologist in the Emergency Department. An Italian Nationwide Epidemiological Survey.” Neurol Sci. 29 (2): 67-75.
[10] Moulin, T., Sablot, D., Vidry, E., Belahsen, F., Berger, E., Rumbach, L., et al. 2003. "Impact of Emergency Room Neurologists on Patient Management and Outcome.” Eur Neurol. 50 (4): 207-14.

[11] Roberts, K., Costelloe, D., Hutchinson, M., and Tubridy, N. 2007. "What Difference Does a Neurologist Make in a General Hospital? Estimating the Impact of Neurology Consultations on In-Patient Care.” Ir J Med Sci. 176 (3): 211-4.

[12] Gómez Ibáñez, A., Irimía, P., and Martínez-Vila, E. 2008. "The Problem of Neurological Emergencies and the Need for Specific Neurology Shifts.” An Sist Sanit Navar 31 (Suppl. 1): 7-13.

[13] Hutchinson, P. J., and Kirkpatrick, P. J. 2002. "Acute Head Injury for the Neurologist.” J Neurol Neurosurg Psychiatry 73: i3-7.

[14] Hanley, D. F., and Hacke, W. 2004. "Critical Care and Emergency Medicine Neurology.” Stroke 35 (2): 365-6.

[15] Moeller, J. J., Kurniawan, J., Gubitz, G. J., Ross, J. A., and Bhan, V. 2008. "Diagnostic Accuracy of Neurological Problems in the Emergency Department.” Can J Neurol Sci. 35 (3): 335-41.

[16] Nuwer, M. R. 2015. “Chronic Care Management Coding for Neurologists.” Neurol Clin Pract. 5 (5): 430-8.

[17] Josephson, S. A., Engstrom, J. W., and Wachter, R. M. 2008. "Neurohospitalists: An Emerging Model for Inpatient Neurological Care.” Ann Neurol. 63 (2): 135-40.

[18] Likosky, D. J. 2009. "Is It Time for Neurohospitalists?" Neurology 72 (9): 859.

[19] Avitzur, O. 2005. "Neurohospitalists: A New Term for a New Breed of Neurologist.” Neurol Today. 5 (10): 44-5.

[20] Likosky, D. J. 2011. "If Not Neurohospitalists, Who? If Not Now, When?” Neurohospitalist 1 (2): 64-6.

[21] Chang, I., and Pratt, R. W. 2012. "Neurohospitalists: An Emerging Subspecialty.” Curr Neurol Neurosci Rep. 12 (4): 481-8.

[22] Douglas, V. C., and Josephson, S, A. 2011. "A Proposed Roadmap for Inpatient Neurology Quality Indicators.” Neurohospitalist 1 (1): 8-15. 